\title{
Human-Computer Interaction and Human Mental Workload: Assessing Cognitive Engagement in the World Wide Web
}

\author{
Luca Longo \\ Department of Computer Science and Statistics, \\ Trinity College Dublin \\ llongo@cs.tcd.ie
}

\begin{abstract}
Assessing the cognitive engagement of a user while seeking and consuming information over the World Wide Web is a key challenge for studying the quality of interactions. Indicators of cognitive engagement are useful for enhancing usability of interfaces, designing adaptable systems but also for analysing user behaviour and performance. For this purpose, we aim to adopt the multifaceted concept of Human Mental Workload, mainly applied in psychology and cognitive sciences, to study individual performance and user engagement in the context of Web. We aim to design a framework in which mental workload can be measured, analysed and explained. This will lead to analysis of individual and mass behaviour, guidelines and recommendation for interaction design, usability of front-end web applications and proposal of adaptive systems.
\end{abstract}

Keywords: Human-Computer Interaction, Human Mental Workload, User Engagement, Artificial Intelligence, Web-mining, Human Factors.

\section{Introduction}

The proliferation of web applications and the explosion of the Internet as an accessible data resource is rapidly changing the way users access, publish, seek and consume information, and knowledge working in general. Interacting with Internet technologies and surfing the WWW is a complex cognitive activity that involves visual, auditory and tactile human modalities rather than physical channels: in these activities human cognition is engaged at multiple stages. Analysing user behaviour while seeking and consuming information over the Web and designing user-centered web applications are becoming key topics in the last few years in the field of HCI. As a consequence, indicators of cognitive engagement are increasingly needed for studying the quality of the user experience while interacting with web resources.

Each action a person performs over the Web can be routinely monitored and stored in web-interaction logs. Existing methodologies for knowledge discovery from such sources are mainly focused on statistical and quantitative analysis. They mainly deal with data such as requested URLs, time properties, the provenance, the type of operating system and browser of a web-user. However, a more fine-grained set of web 
activities such as clicking, scrolling, keyboard usage, mouse movements, that better describe the user's physical interaction with web interfaces, can now be gathered using new Internet technologies.

Although logs turn out to be detailed sources of information, prior research, both academic and industrial, did not prove useful in interpreting logs from a cognitive perspective that links objective web-actions to cognitive theories of task execution. Reading and interpreting interaction $\log s$, from this perspective, is a non-trivial problem: it is extremely difficult to identify users' original intention, goal, degree of attention and interest from these footprints.

Our proposal is to develop an executable framework, inspired by the concept of Human Mental Workload, large-scale applicable in the context of Web, to measure, assess, study and explain users' attention and cognitive engagement [1]. Models of human mental workload, in other fields, have been proven useful in the design of adaptive interfaces and in the study of individual performance. However, the explicit and invasive way of conducting experiments towards indexes of human mental workload, generally laboratory-driven, have not had a large-scale success.

\section{Research Question}

To what extent can the Human-Computer(Web) interaction be automatically captured and interpreted towards automatic assessments of Human Mental Workload?

What are the factors that contribute to define human mental workload in the World Wide Web.

\section{Approach}

Our aim is to define a framework in which the concept of human mental workload can be measured, analysed and explained. The adopted approach is holistic and convergent, and it follows a general progression:

- analytical/empirical exploration of human mental workload characteristics;

- resolving and correlating these characteristics to reliable objective measures;

- definition of an extensible context-aware computational model for the Web;

- $\quad$ validation of the designed prototype with reliable subjective measures.

The research agenda includes the following stages:

1. implementation of a technique that allows non-invasive monitoring of webusers' activity without influencing their experience while consuming information over the World Wide Web;

2. implementation of a technology, large-scale applicable, capable of gathering a more fine-grained corpus of interaction fingerprints such as clicking, scrolling, mouse movements and keyboard usage, closer to the physical human-computer interaction;

3. identification of interaction patterns and development of human mental workload characteristics for the Web; 
4. designing an extensible computational model for aggregating human mental workload characteristics;

5. validation and justification of generated indexes of human mental workload by comparison with existing psychology and cognitive measures [1, 2];

6. application of generated indexes for designing engaging technologies and web applications.

The first 2 stages are aimed at developing a transparent non-invasive technology for gathering detailed human-computer interaction patterns while surfing the Web. The third aims to identify and objectively assess the factors that influence human mental workload in the context of Web. The fourth includes the design of a computational prototype, scenarios-aware, able to aggregate the factors individuated in 3 for a certain web-scenario. The fifth stage is aimed at validating the computational model via comparisons with subjective existing measures, successfully applied in psychology or cognitive science. The last gives practical means to deploying metrics, definitions and techniques for designing adaptable and engaging web applications. The research stages are not independent, rather they are related aspects that inform and influence the entire approach towards the measurement and definition of human mental workload The ongoing work is at stage 3 and 4 . The monitoring technology has been deployed and the human mental workload characteristics along with the parallel definition of the computational model, are investigated.

\section{Sketch of the Solution}

Our framework is composed by 5 functional-based macro-levels. The humancomputer interaction level underlines the process in which users interact with webinterfaces seeking and consuming information. Their interaction is monitored at the technology level: a designed technology aims to mine detailed signs of interaction such as mouse movements, keyboard usage and web-browser events over time. Here a raw interaction log is produced and subsequently consumed at the pre-processing level. Row data is pre-processed using machine learning techniques towards the identification of web-interaction sessions and tasks within each session. Each task is then recognised to be of a certain typology, thanks to an abstract high-level tasktaxonomy. This carries information about task demands in terms of cognitive resources and difficulty. At the computational heuristic level characteristics of human mental workload are individuated, computed and aggregated towards a unique index for a given task. Example of characteristics that may influence the mental workload are task interference, cognitive resources overlapping between multiple tasks, task concurrency, time sharing as suggested by the multiple resource theory of attention proposed by Wickens [3,4]. Individual skills and traits are important characteristics that undoubtedly moderate levels of workload as well as time and richness/control of online activity. The human mental workload characteristics relate to specific scenarios and how they can be calculated by adopting state-of-the-art metrics in psychology, IR or novel metrics. The last application level is represented by the practical use of indexes of human mental workload towards the design of usercentered technologies and user engagement aware web-applications. 


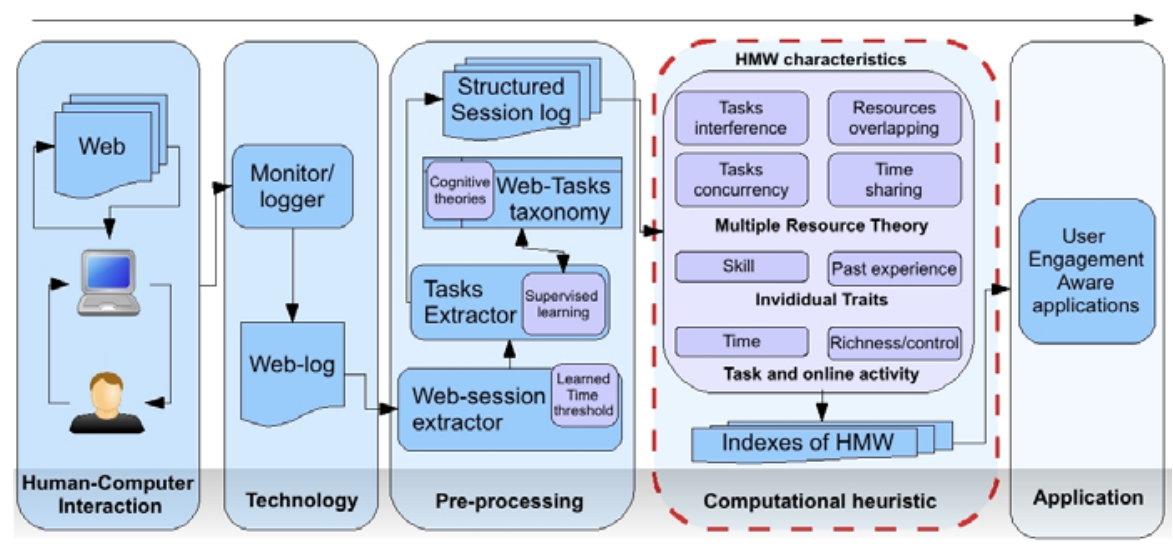

Fig. 1. Anatomy of a framework based on Human Mental Workload assessments

\section{Expected Contribution of the Research}

The research will contribute to the field of Human-Computer Interaction within several areas, proposing a novel large-scale executable paradigm capable of human mental workload automatic assessment in the context of Web. The expected outcomes of the line of research are to produce an applicable framework able to lead to generalisable design recommendations and strategies along with examples and guidelines employable by other researchers or designers. Our contribution will provide reference and case studies for the application of a theory useful for promoting user-engagement aware applications and interactive design contributing to the appreciation and support of design practice in HCI.

\section{References}

1. Kantowitz, B.H.: Development of Nasa-TLX (Task Load Index): Results of Empirical and Theoretical Research. Human Mental Workload 52, 139-183 (1988)

2. O'Brien, H.L., Toms, E.G.: What is user engagement? A conceptual framework for defining user engagement with technology. Journal of the American Society for Information Science \& Technology 59(6), 938-955 (2008)

3. Wickens, D.C., McCarley, J.S.: Applied Attention Theory. CRC, Boca Raton (2008)

4. Wickens, C.D.: Multiple Resource and Performance Prediction. Theoretical Issues in Ergonomics Science 3(2), 159-177 